\title{
On bimodal size distribution of spin clusters in the one- dimensional Ising model
}

\author{
A. Ivanytskyi ${ }^{1, a}$ and V. Chelnokov ${ }^{1}$ \\ ${ }^{1}$ Bogolyubov Institute for Theoretical Physics, Metrologichna str. 14 b, 03143 Kyiv, Ukraine
}

\begin{abstract}
The size distribution of geometrical spin clusters is exactly found for the onedimensional Ising model of finite extent. For the values of lattice constant $\beta$ above some "critical value" $\beta_{c}$ the found size distribution demonstrates the non-monotonic behaviour with the peak corresponding to the size of the largest available cluster. In other words, for high values of the lattice constant there are two ways to fill the lattice: either to form a single largest cluster or to create many clusters of small sizes. This feature closely resembles the well-know bimodal size distribution of clusters which is usually interpreted as a robust signal of the first order liquid-gas phase transition in finite systems. It is remarkable that the bimodal size distribution of spin clusters appears in the one-dimensional Ising model of finite size, i.e. in the model which in thermodynamic limit has no phase transition at all.
\end{abstract}

\section{Introduction}

During the last two decades the experimental studies of phase transitions in finite and even in small systems have inspired a high interest to their rigorous theoretical treatment [2? -4]. One of the main reasons for such an interest is that the nuclear systems which are experimentally studied at intermediate [5-7] and at high [8,9] collision energies have no thermodynamic limit due to the presence of the long range Coulomb interaction and, hence, in a strict thermodynamic sense the phase transition in such systems is not defined. Therefore, the practical need to formulate the reliable experimental signals of phase transformations in the systems consisting of a few hundred or thousands of particles lead researchers to develop of the non-traditional statistical methods which may be suited to small systems [2, 4, 10-12]. One of such directions of research, the concept of bimodality, is based on the old T. L. Hill idea [13] that in a finite system the interface between two pure phases "cost" additional free energy and, hence, their coexistence is suppressed. The practical conclusion coming out of this idea is that the resulting distribution of the order parameter should demonstrate a bimodal behaviour and, hence, each maximum or peak of the bimodal distribution has to be associated with the pure phase $[2,10,13-16]$.

Although in [14] the authors claimed to establish the one-to-one correspondence between the bimodal structure of specially constructed partition of some measurable quantity, known on average, and the properties of the Lee-Yang zeros [17] of this partition in the complex plane of fugacity of this measurable quantity, there appeared certain doubts $[4,12,18]$ about such a strict relation between the

ae-mail: aivanytskyi@bitp.kiev.ua 
bimodal-like mass/charge distributions of nuclear fragments and the liquid-gas nuclear phase transition in finite systems. In particular, the doubts follow from the experimental analysis of high momentum decay channels in nucleus-nucleus collisions [18] and from the exact analytical solutions of the simplified statistical multifragmentation model [19] found for finite [4, 11, 12] and infinite [20] volumes. Two explicit counterexamples suggest that the bimodal mass distribution of nuclear fragments appears in a finite volume analog of gaseous phase [12] with positive surface tension and that it can also occur in the thermodynamic limit at supercritical temperatures [20], if at such temperatures there exists negative surface tension.

Despite the existing counterexamples [12, 20], until now it is unclear, whether the statistical systems without phase transition can, in principle, generate the bimodal size distributions of their constituents for finite volumes. In order to demonstrate that this is, indeed, the case and that the statistical systems in finite volume can generate the bimodal size distributions, here we analytically calculate the size distribution of geometrical spin clusters of the simplest statistical model which has no phase transition, the one-dimensional Ising model [21]. Another principal purpose of this work is to further develop connections between the spin and cluster models respectively.

The cluster models are successfully applied to study phase transitions in simple liquids [22-24], in nuclear matter $[4,11,12,19,20,25]$ and in the quark-gluon plasma phenomenology [26-33]. Their basic assumption is that the properties of the studied system consisting of the elementary degrees of freedom (molecules, nucleons, partons, etc.) can be successfully described in terms of physical clusters (vapor droplets, nucleus, hadrons, etc.) formed by any natural number of the elementary degrees of freedom. Since the cluster models proved to be a successful tool to investigate the phase transition mechanisms both in finite and infinite systems respectively, it seems that the development of an equivalent cluster formulation for the well-known spin models and the determination of the properties of their physical clusters is a theoretical task of high priority. It is necessary to mention that some results on such a connection were already reported in [3], in which a parameterization of the Fisher liquid droplet model [22] was successfully applied to the numerical description of cluster multiplicities of two- and three-dimensional Ising model. However, below we implement this program to the one-dimensional Ising model and directly calculate the size distribution of the geometrical spin clusters from the corresponding partition function.

This work is organized as follows. In the next section we explain the necessary mathematical aspects of calculating the spin cluster size distribution. Then, in Sect. 3 the derived expression is analysed in detail and the physical origin of bimodality in the present model is discussed. The results of our numerical simulations are also presented in this section. Finally, our conclusions and perspectives on further research are summarized in Sect. 4.

\section{Cluster size distribution}

Here we use the geometrical definition of clusters which seems to be the most natural for lattice models. Within this framework monomers, dimers, trimers and so on are built from the neighbouring spins of the same direction. This approach is useful for different lattice systems [3, 34-36]. A high level of its generality allows one to study the SU(2) and SU(3) gauge lattice models in terms of the clusters composed from the Polyakov loops [37-40]. Let us consider the one-dimensional Ising model in which spins are arranged in a linear lattice of size $N$ and they can only be in two states (up or down). Interaction energy of two neighbouring spins is proportional to their product. Therefore, the neighbouring pair of parallel spins contribute $-\epsilon$ to the total energy of the system, whereas the corresponding contribution of the neighbouring pair of antiparallel spins is $\epsilon$. For convenience we choose the following boundary conditions: two edge spins have only a single neighbour which is able to interact with them. 
Evidently, in the present model there are only two types of clusters, i.e. the clusters composed of spins up and of spins down. Hence, for convenience hereafter they are called the up clusters and the down clusters and are marked with the superscripts $\uparrow$ and $\downarrow$, respectively. The cluster multiplicities are defined as their occupancy numbers $n_{k}^{\uparrow}$ and $n_{k}^{\downarrow}$, where $k$ is the cluster size. It is clear that the size of the maximal cluster cannot exceed $N$ and, hence, only the spin configurations obeying the condition $n_{k>N}^{\uparrow}=n_{k>N}^{\downarrow}=0$ can be realized in the considered system. The total number of up and down clusters are, respectively, denoted as $n^{\uparrow} \equiv \sum_{k} n_{k}^{\uparrow}$ and $n^{\downarrow} \equiv \sum_{k} n_{k}^{\downarrow}$. Hereafter, the blind index of cluster size runs over all positive integers. In what follows, the set of all occupancy numbers $\sigma \equiv\left\{n_{k}^{\uparrow}, n_{k}^{\downarrow}\right\}$ is called a microscopic configuration. Each microscopic configuration $\sigma$ of the system defines its total energy, which receives a contribution $\epsilon(1-k)$ from every up or down cluster of size $k$. In addition, there exist the contacts of neighbouring clusters with opposite spins and their number is $n^{\uparrow}+n^{\downarrow}-1$. Evidently, any such contact gives a contribution $\epsilon$ to the system energy. Therefore, using the definitions of $n^{\uparrow}$ and $n \downarrow$ we can cast the total energy of the system for a given microscopic configuration $\sigma$ as

$$
H_{\sigma}=\epsilon\left[\sum_{k=1}^{\infty}(2-k) n_{k}^{\uparrow}+\sum_{k=1}^{\infty}(2-k) n_{k}^{\downarrow}-1\right] \text {. }
$$

Obviously, the total number of spins $N_{\sigma} \equiv \sum_{k}\left(n_{k}^{\uparrow}+n_{k}^{\downarrow}\right) k$ coincides with the lattice size $N$. Since up and down clusters alternate each other, then the only configurations with $n^{\uparrow}-n^{\downarrow}=0, \pm 1$ are realized in the considered system. Hence, taking into account the fact that the total number of cluster permutations is $n^{\uparrow} ! n^{\downarrow} ! / \prod_{k} n_{k}^{\uparrow} ! n_{k}^{\downarrow}$ ! we write the degeneracy factor of the microscopic configuration $\sigma$ as

$$
G_{\sigma}=\delta_{N, N_{\sigma}}\left[2 \delta_{n^{\downarrow}, n^{\uparrow}}+\delta_{n^{\downarrow}+1, n^{\uparrow}}+\delta_{n^{\downarrow}, 1+n^{\uparrow}}\right] n^{\uparrow} ! n^{\downarrow} ! / \prod_{k=1}^{\infty} n_{k}^{\uparrow} ! n_{k}^{\downarrow} !,
$$

where $\delta$ is the Kronecker delta-symbol. Note that every configuration with $n^{\downarrow}=n^{\uparrow}$ should have a degeneracy 2, since it can be "constructed" only from the configurations $n^{\downarrow}+1=n^{\uparrow}$ and $n^{\downarrow}=1+n^{\uparrow}$ by combining one of their outer clusters with any other cluster of the spin sign which coincides with the one of the outer cluster.

The $q^{\text {th }}$ statistical moment of the down cluster occupancy number we define as

$$
\left\langle n_{l}^{\downarrow q}\right\rangle=\frac{1}{Z} \sum_{\sigma}\left[n_{l}^{\downarrow}\right]^{q} G_{\sigma} e^{-\frac{H_{\sigma}}{T}+a \beta\left(N_{\sigma}-N\right)},
$$

where $T$ is the temperature $\beta=\epsilon / T$ is the lattice constant. A summation over the configurations $\sigma$ in (3) should be understood as a summation over all non-negative integers $n_{k}^{\uparrow}$ and $n_{k}^{\downarrow}$. The dimensionless parameter $a$ is introduced in (3) for the convenience of analytical calculations and its value is chosen to satisfy the conditions $\beta(a+1)<0$ and $\beta a<-\ln (2 \cosh \beta)$. However, this parameter does not affect any observable due to the condition $N_{\sigma}=N$ provided by the Kronecker delta-symbols in Eq. (2).

Note that $q=0$ yields the partition function $Z$, whereas a symmetry between up and down spins leads to the equality $\left\langle n_{l}^{\uparrow q}\right\rangle=\left\langle n_{l}^{\downarrow q}\right\rangle$. Using Eqs. (1), (2), the definition of $N_{\sigma}$ and the integral representation of the Kronecker delta-symbol $\delta_{r, s}=\int_{0}^{2 \pi} \frac{d \alpha}{2 \pi} e^{i \alpha(r-s)}$ and of the factorials $n^{\uparrow \downarrow} !=\int_{0}^{\infty} \frac{d z}{e^{z}} z^{n^{\uparrow \downarrow}}$ we get

$$
\begin{aligned}
\left\langle n_{l}^{\downarrow q}\right\rangle= & \frac{e^{\beta(1-a N)}}{Z} \int_{0}^{2 \pi} \frac{d \psi}{2 \pi} e^{i \psi N} \int_{0}^{2 \pi} \frac{d \phi}{2 \pi}\left(2+e^{i \phi}+e^{-i \phi}\right) \\
& \cdot \int_{0}^{\infty} d x e^{-x} \prod_{k=1}^{\infty} \sum_{n_{k}^{\uparrow}=0}^{\infty} \frac{\left(x e^{\beta(k(a+1)-2)-i \phi-i \psi k}\right)^{n_{k}^{\uparrow}}}{n_{k}^{\uparrow} !} \int_{0}^{\infty} d y e^{-y} \prod_{k=1}^{\infty} \sum_{n_{k}^{\downarrow}=0}^{\infty} \frac{\left(y e^{\beta(k(a+1)-2)+i \phi-i \psi k}\right)^{n_{k}^{\downarrow}}}{n_{k}^{\downarrow} !} n_{l}^{\downarrow q} .
\end{aligned}
$$

Now the summations over $n_{k}^{\uparrow}$ and $n_{k \neq l}^{\downarrow}$ in (4) can be performed trivially giving the corresponding exponential functions. In what follows we are mainly interested in finding the statistical moments 
of $n_{l}^{\downarrow}$ of the zeroth and first order. For $q=\{0 ; 1\}$ the summation over $n_{l}^{\downarrow}$ generates an exponential function multiplied by the factor $y^{q} \exp (q \beta(l(a+1)-2)+i q \phi-i q \psi l)$. Then, each product over $k$ in (4) can be carried out, since it is equivalent to a summation of decreasing geometric progressions in the exponential. In addition, the chosen range of $a$ provides a convergence of the integrals over $x$ and $y$ variables due to negative real part of these exponential functions. Hence, for $q=0$ and 1 one can easily find

$$
\left\langle n_{l}^{\downarrow}\right\rangle=\frac{e^{\beta(q(l-2)+1)}}{Z} \oint_{|\zeta|=e^{-a \beta}} \frac{d \zeta \zeta^{N-q l-1}}{2 \pi i} \oint_{|\xi|=1} \frac{d \xi\left(2+\xi+\xi^{-1}\right) \xi^{q}}{2 \pi i\left(\xi-\frac{e^{-\beta}}{\zeta-e^{\beta}}\right)\left(1-\frac{\xi e^{-\beta}}{\zeta-e^{\beta}}\right)^{q+1}},
$$

where the real variables of integration $\phi$ and $\psi$ are now changed to the complex ones $\xi=e^{i \phi}$ and $\zeta=e^{i \psi-a \beta}$. The integrand with respect to the variable $\xi$ in Eq. (5) contains three poles. The first one $\xi=0$ exists for $q=0$ only. However, the contribution of this pole does not produce any singularity with respect to the $\zeta$ variable and therefore it can be safely neglected. The second pole existing at any value of $q$ corresponds to $\xi=e^{\beta}\left(\zeta-e^{\beta}\right)$. It does not contribute to $\left\langle n_{l}^{\downarrow}\right\rangle$ since it is located out of the contour $|\xi|=1$ due to the chosen value of the regularization parameter $a$. Thus, accounting only for the simple pole $\xi=e^{-\beta} /\left(\zeta-e^{\beta}\right)$ we obtain

$$
\left\langle n_{l}^{\downarrow}\right\rangle=\frac{e^{\beta(q(l-3)+2)}}{Z} \oint_{|\zeta|=e^{-a \beta}} \frac{d \zeta}{2 \pi i} \frac{\zeta^{N-q l-1}(\zeta-2 \sinh \beta)^{1-q}\left(\zeta-e^{\beta}\right)^{q+1}}{(\zeta-2 \cosh \beta)^{q+1}} .
$$

This expression clearly shows that for $q=1$ and $l=N$ the integral function has a specific simple pole $\zeta=0$ which, as we discuss below, is responsible for the non-monotonic behaviour of $\left\langle n_{l}^{\downarrow}\right\rangle$.

Taking $q=0$ we immediately reproduce the well-known partition function of the one-dimensional Ising model with free edges [21], i.e.

$$
Z=e^{2 \beta} \oint_{|\zeta|=e^{-a \beta}} \frac{d \zeta \zeta^{N-1}(\zeta-2 \sinh \beta)\left(\zeta-e^{\beta}\right)}{2 \pi i(\zeta-2 \cosh \beta)}=2(2 \cosh \beta)^{N-1}
$$

For $l \leq N$ the average occupancy number of the down clusters is obtained from Eq. (6) for $q=1$

$$
\left\langle n_{l \leq N}^{\downarrow}\right\rangle=\frac{e^{\beta(1-l)}}{Z} \oint_{|\zeta|=e^{-a \beta}} \frac{d \zeta \zeta^{N-l-1}\left(\zeta-e^{\beta}\right)^{2}}{2 \pi i(\zeta-2 \cosh \beta)^{2}}=\frac{\delta_{N, l}+2 e^{-2 \beta}+(N-l+1) e^{-4 \beta}}{2\left(1+e^{-2 \beta}\right)^{l+1}},
$$

whereas $\left\langle n_{l>N}^{\downarrow}\right\rangle=0$. Note that $\left\langle n_{l}^{\uparrow}\right\rangle=\left\langle n_{l}^{\downarrow}\right\rangle$ for all $l$. The direct calculation based on this expression demonstrates that $\sum_{l} l\left\langle n_{l}^{\uparrow}\right\rangle=\sum_{l} l\left\langle n_{l}^{\downarrow}\right\rangle=N / 2$ which is an obvious consequence of the symmetry between the spins up and down, if the external magnetic field is absent. It is necessary to note that the cluster number density $\rho_{l}=\left\langle n_{l}^{\downarrow}\right\rangle / N$ of a similar model was investigated in Ref. [41]. In fact, the authors of Ref. [41] studied the one-dimensional lattice gas model using the maximum term method. Since such a method is, strictly speaking, valid in the thermodynamic limit only, the results of Ref. [41] are not applicable to the truly finite systems. This is the reason why the expression for $\rho_{l}$ derived in [41] does not contain a term which is proportional to the Kronecker $\delta$-function $\delta_{N, l}$ and which is important for finite systems. At the same time, in the limit of an infinite lattice $(N \rightarrow \infty) \mathrm{Eq}$. (8) gives us $\rho_{l}=e^{-4 \beta} / 2\left(1+e^{-2 \beta}\right)^{l+1}$. The latter expression coincides with Eqs. (19) and (20) of Ref. [41] obtained for equal volume fractions of clusters of both kinds $\left(\theta_{1}=\theta_{0}=1 / 2\right.$ in the notations of [41]), if in the notations of Ref. [41] the lattice constant $\beta$ of this work is defined as $\beta=\epsilon / 4 k_{B} T$. Here $k_{B}$ is the Boltzmann constant and $T$ is the temperature.

An explicit expression (8) for $\left\langle n_{l}^{\downarrow}\right\rangle$ allows us to find the size distribution function of clusters $\omega_{l}$ which is proportional to their average occupancy number. Since the size distribution functions are the same for both kinds of clusters, we do distinguish them. The normalized distribution $\omega_{l}$ is obtained 
from the condition that the total probability to find up (or down) cluster of any size is $1 / 2$, i.e. $\sum_{l} \omega_{l}=$ $1 / 2$. This condition follows from the symmetry between up and down spins. Hence, we obtain

$$
\omega_{l \leq N}=\frac{\delta_{N, l}+2 e^{-2 \beta}+(N-l+1) e^{-4 \beta}}{2\left(1+N e^{-2 \beta}\right)\left(1+e^{-2 \beta}\right)^{l}},
$$

and $\omega_{l>N}=0$. From Eq. (9) one can see that $\omega_{l}$ has not only the exponential part $\left(1+e^{-2 \beta}\right)^{-l}$, but also it includes the factor which is linear in $l$. The power part of the size distribution functions is known in several statistical models of cluster type. Traditionally it is taken into account by the Fisher topological exponent $\tau$ as $l^{-\tau}[4,22,25,28]$. However, the linear $l$-part of the size distribution function (9) disappears in the thermodynamic limit $N \rightarrow \infty$, since in this limit one finds $\omega_{l} \rightarrow e^{-2 \beta}\left(1+e^{-2 \beta}\right)^{-l} / 2$. Hence, it is interesting to analyse the distribution $\omega_{l}$ of other lattice models in order to find possible restrictions on values of the Fisher index $\tau$.

\section{Bimodality manifestation}

The growing interest in a bimodality is caused by the widespread belief that it can serve as a robust signal of the first order phase transition in finite systems. As it was mentioned above this concept is based on T. L. Hill's idea [13], that each peak of the bimodal distribution is associated with a pure phase. However, this idea cannot be sufficiently justified, since in finite systems the analogue of mixed phase is not just a mixture of two pure phases [4, 11, 12]. According to exact analytical solutions found for several cluster models in finite systems $[4,11,12]$ the finite volume analogue of mixed phase is represented by a combination of an analogue of gaseous phase, which is stable, and some even number of different metastable states. The number of metastable states is determined by thermodynamic parameters, but their identification with the help of a single peak (or maximum) of the size distribution function does not seem to be a well defined procedure $[4,11,12]$.

Having at hand an explicit expression for cluster size distribution we are able to answer the question what is the reason for a bimodal behaviour. From Eq. (9) it follows that $\omega_{l}=\delta_{N, l} / 2$ for finite $N$ and for $\beta \rightarrow \infty$ (a ferromagnetic system at zero temperature), whereas the limit $\beta \rightarrow-\infty$ (an antiferromagnetic system at zero temperature) generates $\omega_{l}=\delta_{1, l} / 2$ for finite $N$. Therefore, we conclude that at some intermediate value of the lattice constant $\beta$ the monomer dominant regime should switch to the regime of dominance of the cluster of maximal size. This switching is characterized by the comparable probabilities to find the monomers (dimers, trimers, etc.) and the cluster of maximal size and, hence, at some value of $\beta$ the distribution function $\omega_{l}$ becomes a non-monotonic one.

A direct inspection of Eq. (9) shows that for $l<N$ and finite $N$ the derivative $\frac{\partial \omega_{l}}{\partial l}$ is always negative, i.e. $\frac{\partial \omega_{l}}{\partial l}<0$ and, hence, at this interval of cluster sizes the function $\omega_{l}$ is monotonically decreasing. Let us show that the cluster size distribution can be non-monotonic due to presence of the Kronecker delta-symbol $\delta_{N, l}$. At $l=N$ the cluster size distribution increases if the inequality $\omega_{N-1}<\omega_{N}$ holds or, equivalently, if $\beta>\beta_{c} \equiv \ln 2 / 2$. Thus, at the values of the lattice constant greater than $\beta_{c}$ the cluster size distribution becomes non-monotonic. Inequality $\beta>\beta_{c}$ can only be fulfilled for $\epsilon>0$. This means that the non-monotonicity of $\omega_{l}$ in the one-dimensional Ising model can only appear in the ferromagnetic case. It is necessary to stress that the value of $\beta_{c}$ does not depend on $N$ or $\epsilon$ and therefore it is a universal constant. Thus, in the ferromagnetic case the found cluster distribution function $\omega_{l}$ is non-monotonic (bimodal) for any size of the lattice and any value of the spin coupling. However, we should note that the bimodal behaviour of the cluster size distribution is washed out in the thermodynamic limit $N \rightarrow \infty$, since in this case $\omega_{N}-\omega_{N-1} \rightarrow 0$ for all finite values of the lattice constant $\beta$. This fact explains the reason why the bimodality in the one-dimensional lattice gas model was not observed in Ref. [41]. Since in Ref. [41] the number density of clusters was studied in the thermodynamic limit, then, evidently, all finite size effects were washed out in such a treatment. We 

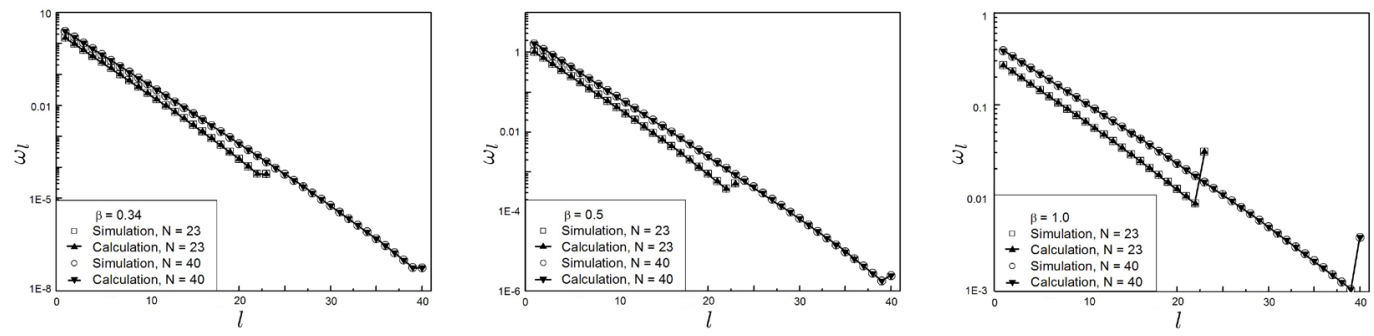

Figure 1. Cluster size distribution calculated using Eq. (9) (triangles) and found numerically for $N=23$ and $N=40$ (squares and circles) for three values of the lattice constant $\beta$. For $\beta=0.34<\beta_{c}$ (upper panel) $\omega_{l}$ monotonically decreases with the cluster size growth, while for $\beta=0.5>\beta_{c}$ (middle panel) and $\beta=1.0>\beta_{c}$ (lower panel) it has a maximum at $l=N$.

would like, however, to mention that the bimodal distribution of energy [42] and the one of the largest cluster size [43] were recently found in the five-dimensional Ising model with the cyclic boundary conditions. However, their physical origin was not discussed in Refs. [42, 43].

The mathematical reason of the non-monotonic behaviour of the cluster size distribution in the present model is clear now. Although in this model the bimodality phenomenon appears as the finite size effect, we would like to add a few words on its substantial origin. For this purpose let us consider an auxiliary infinite lattice with the cluster distribution function $\tilde{\omega}_{l}$, which for all $l$ obeys the inequalities $N<l<l_{0}$, is defined as

$$
\tilde{\omega}_{l}=\frac{2 e^{-2 \beta}+(N-l+1) e^{-4 \beta}}{2\left(1+N e^{-2 \beta}\right)\left(1+e^{-2 \beta}\right)^{l}}
$$

where $l_{0}=N+1+2 e^{2 \beta}$ provides the non-negative value of $\tilde{\omega}_{l_{0}}$. For $\beta \gg 1$ the distribution (10) describes clusters of almost all sizes (up to $l_{0} \gg N$ ) on an equal footing. Observe that in Eq. (10) the quantity $N$ is treated as a finite parameter. Suppose now that we randomly choose in this infinite lattice the infinite amount of intervals of the length $N$ each. It is clear that the size distribution of small clusters will be given by Eq. (10), whereas the clusters with $l>N$ will increase the occupancy number of the cluster of maximal size (they will also contribute to the smaller clusters, but this fact is already taken into account in (9)). This can be easily seen, if one sums up all values of $\tilde{\omega}_{l}$ for $l>N$

$$
\sum_{l=N+1}^{\infty} \tilde{\omega}_{l}=\frac{1}{2\left(1+N e^{-2 \beta}\right)\left(1+e^{-2 \beta}\right)^{N}}=\omega_{N}-\tilde{\omega}_{N} .
$$

Although in the sum above the terms with $l>l_{0}$ are negative, their contribution is practically negligible due to the adopted assumption that $\beta \gg 1$. The result of summation is nothing else than the term in $\omega_{N}$ of Eq. (9) which is proportional to the Kronecker delta-symbol. Eq. (11) shows that in a finite system the clusters of size larger than the lattice size are "condensing" into the largest cluster of size $N$, whereas their probabilities $\tilde{\omega}_{N<l<l_{0}}$ are "absorbed" into $\omega_{N}$.

One may wonder, what is the relation between the non-monotonic size distribution (9) and, for example, the size distribution of nuclear fragments [5-7, 25] (or other cluster size distributions studied experimentally [1-3]) which usually has a wide maximum of the largest cluster instead of a peak. It is necessary to note that in real experiments the size of a studied system, i.e. the number of spins $N$ in our case, is not a constant, but it fluctuates from sample to sample around some average value. Therefore it is possible that in the real experiments such fluctuations lead to a widening of the sharp peak and it becomes a maximum. Such a procedure is well described and applied to the nuclear fragments in [12]. 
In order to verify the occurrence of bimodality in the one-dimensional Ising model we present here the results of our numerical simulations for the lattice sizes $N=23$ and $N=40$. These simulations were made using the Swendsen-Wang algorithm. For each run the first $5 \cdot 10^{5}$ lattice configurations were discarded to ensure a complete thermalization, and the next $5 \cdot 10^{7}$ lattice measurements were made while discarding every 5 lattice configurations between the measurements. For each measurement the total expectation of the number of clusters of all sizes was calculated, then the jackknife error analysis was used to obtain the error estimate of the mean values obtained.

Our numerical study includes 14 values of the lattice constant in the range from 0 to 2.5 . We would like to stress that the coincidence of distribution functions simulated numerically and calculated according to formula (9) is perfect. In Fig. 1 the cluster size distribution is shown for three values of the lattice constant $\beta$. For $\beta=0.34<\beta_{c}$ the cluster size distribution is monotonic for both values of $N$. At the same time for the values of the lattice constant above the "critical" one, i.e. for $\beta=0.5>\beta_{c}$ and for $\beta=1.0>\beta_{c}$, one can clearly see the bimodal distribution in Fig. 1. It is also seen that bimodality is enhanced with the lattice constant increasing. We have to remind once again that the present model does not have any phase transition in the thermodynamic limit. Hence, the one-dimensional Ising model gives an explicit counterexample to treating bimodality as a signal of phase transition in finite systems.

\section{Conclusions and perspectives}

In this work we found an analytical solution of the one-dimensional Ising model in terms of the geometrical clusters composed of the neighbouring spins of the same direction. It is clear that the present formulation is an important step towards establishing a firm connection between the lattice spin systems and the statistical models of cluster type. The developed approach allows us to exactly calculate the size distribution of spin clusters for finite and infinite lattice sizes. Using the exact formulae we showed that for finite size of the lattice the one-dimensional Ising model has a bimodal size distribution of clusters for $\beta>\ln 2 / 2$. This nontrivial result was overlooked in Ref. [41] because its authors employed the asymptotic method which is well suited for large systems.

Moreover, we explicitly show that the bimodal size distribution of spin clusters in the present model is a finite size effect which appears due to a "condensation" into a largest cluster of the clusters whose size exceeds the lattice extent. Such a "condensation" to the maximal cluster leads to the non-monotonic size distribution at sufficiently large values of the lattice constant in ferromagnetic case only. The obtained result is valid for all sizes of the lattice, but in the thermodynamic limit it is washed out. Such a result provides us with an explicit counterexample to the widely spread belief about an exclusive role of bimodality as a signal of phase transition in finite systems [2, 13-16]. Our criticism is in full coherence with the results of Refs. $[4,12,18]$. The developed formalism considers the simplest exactly solvable lattice model, but it would be interesting to apply it to more realistic physical systems.

Acknowledgments. The authors appreciate the fruitful discussions with K. A. Bugaev, D. R. Ollinychenko and V. K. Petrov and their valuable comments. Also the authors are thankful to P. H. Lundow and K. Markstrøm for pointing out their original results. The work of A. I. I. is supported in part by the National Academy of Sciences of Ukraine Grant of GRID simulations for high energy physics. 


\section{References}

[1] D. H. E. Gross, Phys. Rep. 279, 119 (1997).

[2] Ph. Chomaz, F. Gulminelli and V. Duflot, Phys. Rev. E, 64, 046114 (2001).

[3] L. G. Moretto et al., Phys. Rev. Lett., 94, 202701 (2005).

[4] K. A. Bugaev, Phys. Part. Nucl., 38, 447 (2007).

[5] J. Natowitz et al., Phys. Rev. C, 65, 034618 (2002).

[6] Pichon M. et al. (INDRA and ALADIN Collaborations), Nucl. Phys. A, 779, 267 (2006).

[7] E. Bonnet et al. (INDRA and ALADIN Collaborations), Phys. Rev. Lett., 103, 072701 (2009).

[8] S. Pratt, Physics 1, 29 (2008)

[9] F. Karsch, PoSCPOD, 2013, 046 (046).

[10] F. Gulminelli, Ph. Chomaz, Al. H. Raduta, and Ad. R. Raduta, Phys. Rev. E, 64, 046114 (2001).

[11] K. A. Bugaev, Acta. Phys. Polon., B 36, 3083 (2005).

[12] K. Bugaev, A. Ivanytskyi, V. Sagun, D. Oliinychenko, Phys. Part. Nucl. Lett., 10, 832 (2013).

[13] T. L. Hill, Thermodynamics of small systems, Dover, New York (1994).

[14] Ph. Chomaz and F. Gulminelli, Physica A, 330, 451 (2003).

[15] F. Gulminelli, Ann. Phys. Fr., 29, 6 (2004).

[16] F. Gulminelli, Nucl. Phys. A, 791, 165 (2007).

[17] C. N. Yang and T. D. Lee, Phys. Rev., 87, 404 (1952).

[18] O. Lopez, D. Lacroix, and E. Vient, Phys. Rev. Lett., 95, 242701 (2005).

[19] S. Das Gupta and A. Z. Mekjian, Phys. Rev. C, 57, 1361 (1998).

[20] V. V. Sagun, A. I. Ivanytskyi, K. A. Bugaev, I. N. Mishustin, Nucl. Phys. A, 924, 4, 24 (2014).

[21] E. Ising, Z. Phys., 31, 253 (1925).

[22] M. E. Fisher, Physics, 3, 255 (1967).

[23] A. Dillmann and G. E. Meier, J. Chem. Phys. 94, 3872 (1991).

[24] A. Laaksonen, I. J. Ford, and M. Kulmala, Phys. Rev. E 49, 5517 (1994).

[25] J. P. Bondorf et al., Phys. Rep., 257, 131 (1995).

[26] J. I. Kapusta J. I., Phys. Rev. D, 23, 2444 (1981).

[27] M. I. Gorenstein, G. M. Zinovjev and V. K. Petrov, Phys. Lett. B, 106, 327 (1981).

[28] K. A. Bugaev, Phys. Rev. C, 76, 014903 (2007).

[29] K. A. Bugaev, V. K. Petrov and G. M. Zinovjev, Phys. Part. Nucl. Lett., 9, 3, 238 (2012).

[30] K. A. Bugaev et al., Phys. Atom. Nucl., 75, 6, 707 (2012).

[31] I. Zakout, C. Greiner, J. Schaffner-Bielich, Nucl. Phys., A 781, 150 (2007).

[32] I. Zakout, C. Greiner, Phys. Rev. C, 78, 034916 (2008).

[33] L. Ferroni and V. Koch, Phys. Rev. C, 79, 034905 (2009).

[34] L. G. Moretto et al., Phys. Rev. C, 68,1602 (2003) and references therein.

[35] S. Fortunato and H. Satz, Phys. Lett. B, 475, 311 (2000).

[36] S. Fortunato et. al, Phys. Lett. B, 502, 321 (2001).

[37] C. Gattringer, Phys. Lett. B, 690, 179 (2010).

[38] C. Gattringer and A. Schmidt, JHEP, 1101, 051 (2011).

[39] G. Endrodi, A. Schäfer and J. Wellnhofer, arXiv:1506.07698 [hep-lat].

[40] A. I. Ivanytskyi et. al., Nucl. Phys. A 960, 90 (2017).

[41] M. B. Yilmaz and F. M. Zimmermann, Phys. Rew. E, 71, 026127 (2005).

[42] Lundow P. H. and Markstrøm K., Nucl. Phys. B, 845, 120 (2011).

[43] Lundow P. H. and Markstrøm K., Phys. Rew. E, 91, 022112 (2015). 